\title{
Precision phase control of an ultrawide-bandwidth femtosecond laser: a network of ultrastable frequency marks across the visible spectrum
}

\author{
Jun Ye, John L. Hall, and Scott A. Diddams* \\ JILA, University of Colorado and National Institute of Standards and Technology, Boulder, Colorado 80309-0440
}

Received June 30, 2000

\begin{abstract}
We demonstrate that the stability of the current optical frequency comb generated by a Kerr-lens mode-locked femtosecond laser is limited by the microwave reference used for phase locking the comb spacing. Hence we implement precision frequency/phase control of the entire comb to the fundamental and second-harmonic frequencies of a stable cw laser without any external microwave reference. The stability of a cw iodinestabilized laser is transferred to millions of comb lines (with an instability of $3 \times 10^{-13}$ ) covering more than one octave of the optical frequency spectrum. In addition, the mode spacing of the comb can be used as a stable microwave frequency derived directly from a stable optical oscillator. (c) 2000 Optical Society of America

OCIS codes: $230.4910,320.7090,120.3930,190.2620,220.4840$.
\end{abstract}

A revolutionary advance in optical frequency-based high-resolution spectroscopy and precision metrology has resulted from the recent introduction of ultrawide-bandwidth optical combs generated by ultrafast lasers. ${ }^{1,2}$ The initial demonstration has proved that the uniformity and accuracy of comb spacing reach a level of $1 \times 10^{-17} .{ }^{1}$ The frequency of any comb line can thus be expressed by an integer multiple of mode spacing $\Delta$ (the pulse-repetition rate) plus an offset frequency $\delta$ that arises from the difference between phase and group velocities of the pulses in the laser cavity. With the comb bandwidth extending an entire optical octave, this offset frequency can be determined and controlled to any preassigned value, thus stabilizing the relative phase between the pulse envelope and the optical carrier. ${ }^{3}$ One can carry out precise optical frequency measurements simply by counting the mode orders of the comb. ${ }^{1,2}$ A single-step phase coherent link between optical frequencies and microwave standards, which is a great simplification of the traditional frequency chains based on the principle of harmonic synthesis, is now realized. ${ }^{2}$

It appears fitting that such an attractive measurement capability has arrived at a time when optical frequency standards based on a single ion or on a collection of cold atoms are emerging as potentially the most stable kinds of clocks. ${ }^{4}$ The unrivaled resolution of the recent observation of a $6-\mathrm{Hz}$ resonance linewidth of a single $\mathrm{Hg}^{+}$transition at $282 \mathrm{~nm}^{4}$ will allow unprecedented stability near $1 \times 10^{-15}$ and potential accuracy below $1 \times 10^{-17}$. Measurement of such ultrastable optical frequency standards at these levels requires exceptional, nearly unattainable, frequency stability of the microwave/radio-frequency standards that are used to set the value of $\Delta$. Thus it appears advantageous to use the femtosecond comb to transfer the phase coherence from the optical spectrum to the rf domain instead. In fact, such an arrangement would constitute the necessary clockwork for a primary atomic clock based on an optical transition.
Beyond capitalizing on the high stability of optical standards, the process of frequency division is intrinsically a better technique for transferring clock signals because it avoids the problem of multiplicative accumulation of phase noise. ${ }^{5}$ In this Letter we show

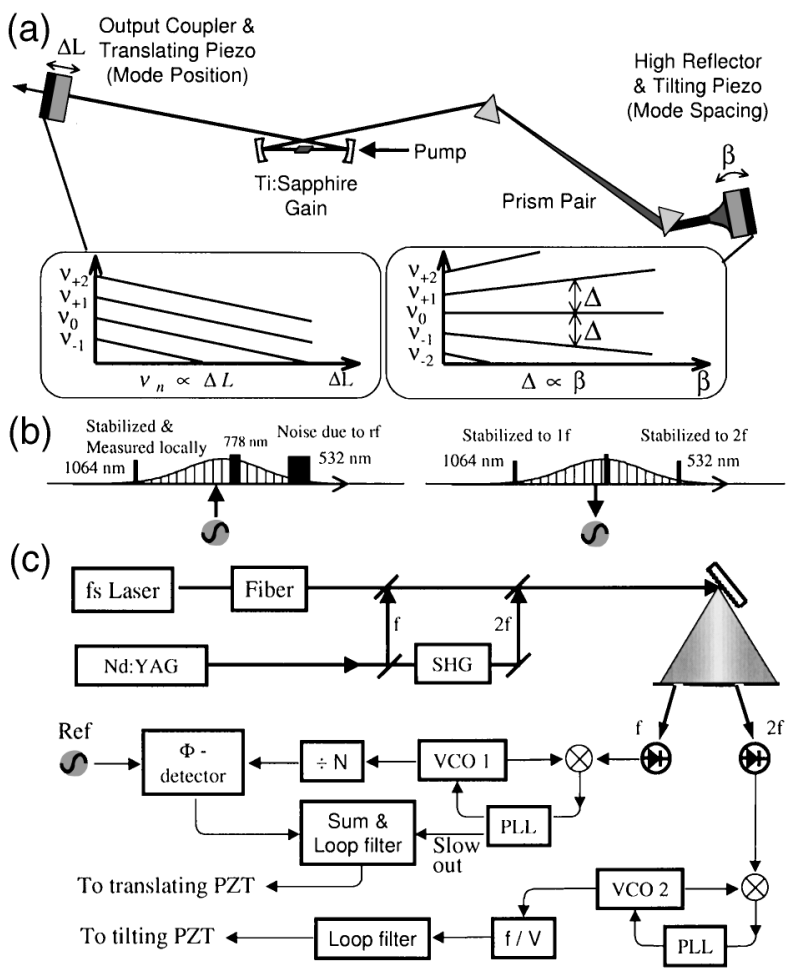

Fig. 1. (a) Ti:sapphire-based Kerr-lens mode-locked femtosecond laser. The translating and tilting PZT's used for frequency/phase stabilization of the laser are also shown. (b) Comparison of the two schemes: (left) using a rf clock to stabilize the comb spacing and (right) generating a rf signal from an optical standard. (c) Implementation of the frequency-phase lock system for the femtosecond laser. SHG, second-harmonic generator; f/v, frequency-voltage converter; other abbreviations defined in text. 
the preliminary implementation of such a clockwork mechanism. A by-product is an optical comb network that spans the whole visible spectrum with millions of frequency marks, all of which are stable at levels of $1-100 \mathrm{~Hz}$ with a $1-\mathrm{s}$ measurement time.

The basic experimental scheme is displayed in Fig. 1. Figure 1(a) shows the Kerr-lens mode-locked femtosecond laser producing $100-\mathrm{MHz}$ repetition-rate pulse trains of $10-\mathrm{fs}$ pulses. Frequency/phase stabilization of this ultrafast laser is implemented with two piezoelectric transducers (PZT's). ${ }^{6}$ The translating PZT varies the cavity length and thus moves all the comb modes together. The tilting PZT controls the intracavity dispersion and thus the comb mode spacing. The issue of orthogonalization of the two control elements is discussed below. To provide coverage of an entire optical octave, we further broaden the bandwidth of the comb by launching the pulse train from the laser into a microstructure fiber. ${ }^{7}$ Figure 1(b) compares the two methods of comb frequency stabilization. At the left our first approach is shown, in which $\Delta$ is phase locked to a $\mathrm{rf}$ reference signal while one comb line is locked to a cw laser. At the right we show our current scheme, in which two comb components, one at each end of the comb spectrum, are stabilized to a $\mathrm{cw}$ reference laser.

Figure 1(c) illustrates the implementation of the phase lock system for the femtosecond laser. An ultrastable cw laser and its frequency-doubled output are used to provide optical references for two comb components. The $\mathrm{cw}$ laser is a Nd:YAG laser at $1064 \mathrm{~nm}$ with its second harmonic at $532 \mathrm{~nm}$. The iodine-stabilized Nd:YAG laser has a frequency stability of $5 \times 10^{-14}$ at 1 -s averaging time, reaching $4 \times 10^{-15}$ at 700 s. $^{8}$ High-speed photodiodes are used to detect simultaneously the two heterodyne beat signals, one between the Nd:YAG laser and its corresponding comb line and the other between the doubled $\mathrm{Nd}$ :YAG laser and one of its neighboring comb lines. High-resolution dispersion systems help to reduce the number of comb components that are received by the detectors to minimize detection noise. The typical signal-to-noise ratio of the beat at 1064 and $532 \mathrm{~nm}$ is $\sim 20 \mathrm{~dB}$ in a $100-\mathrm{kHz}$ bandwidth. This signal-to-noise ratio permits robust phase locking (PLL) between the beat signals and the voltage-controlled tracking oscillators (VCO's), leading to accurate and amplified representation of the actual beat signals. The beat signal at $1064 \mathrm{~nm}$ is frequency prescaled $(\div 256)$ before its phase is compared with a stable rf oscillator. The resultant phase error, along with the slow error correction from the VCO1 tracking process, is used to phase lock this comb component at $1064 \mathrm{~nm}$ to the $\mathrm{cw}$ laser via the translating PZT. The comb component at $532 \mathrm{~nm}$ is frequency locked to the doubled Nd:YAG laser by use of a precision frequency-to-voltage converter for error-signal generation and the tilting PZT for frequency correction.

To illustrate the importance of the new approach in which two comb lines are independently stabilized to cw lasers, we go back to the first scheme shown in Fig. 1(b). In that case we also detect the optical beats at both 1064 and $532 \mathrm{~nm}$, but only the 1064-nm beat is locked to the $\mathrm{cw}$ laser via the translating PZT. The $532-\mathrm{nm}$ beat is used only for diagnostic purposes. Another degree of freedom associated with the comb motion is the mode spacing $\Delta$. To control $\Delta$ we detect a portion of the pulse train with a fast photodiode and phase lock the 100th harmonic of $\Delta$ to a stable microwave source at $10 \mathrm{GHz}$ with the aid of the tilting PZT in the laser cavity. The internal clock of the microwave generator is referenced to a rubidium $\mathrm{rf}$ standard with an (in)stability of $5 \times 10^{-12}$ at a 1 -s averaging time. Figure 2 shows the beat results of such an arrangement. With a simple frequency lock, the beat frequency at $1064 \mathrm{~nm}$ shows a standard deviation of $83 \mathrm{~Hz}$ at a 1-s averaging time. The beat signal at $532 \mathrm{~nm}$, which is $282 \mathrm{THz}$ away from the $1064-\mathrm{nm}$ beat, displays a substantially larger frequency fluctuation, with a standard deviation of $1.9 \mathrm{kHz}$ at $1-\mathrm{s}$, limited seemingly directly by the stability of the rubidium clock. Another cw laser, at $778 \mathrm{~nm}$, stabilized on a rubidium two-photon transition, is also used, and we detect its beat against a corresponding comb line there. The result, with a standard deviation of $690 \mathrm{~Hz}$ at $1 \mathrm{~s}$, is shown in Fig. 2. The fractional instabilities $\left(3 \times 10^{-13}\right.$ for $1064 \mathrm{~nm}, 1.8 \times 10^{-12}$ for $778 \mathrm{~nm}$, and $3.4 \times 10^{-12}$ for $532 \mathrm{~nm}$ ) indicated in Fig. 2 are calculated from the ratios of the frequency standard deviations over their corresponding optical carriers. However, if the frequency noise is compared with the measured frequency gap, such as $690 \mathrm{~Hz}$ over $104 \mathrm{THz}$ $(1064$ to $778 \mathrm{~nm})$ or $1.9 \mathrm{kHz}$ over $282 \mathrm{THz}(1064$ to $532 \mathrm{~nm}$ ), the fractional instability is $6 \times 10^{-12}$, very near the microwave stability limit. The suspicion that the $\mathrm{rf}$ reference used to stabilize $\Delta$ is the chief source of noise in those optical beats (away from $1064 \mathrm{~nm}$ ) is further confirmed by use of a different rubidium clock, with worse stability, to stabilize $\Delta$. The result is that the frequency noise of the optical beat at $532 \mathrm{~nm}$ increases by more than a factor of 2 .

An improvement of the system is of course the use of a more stable $r f$ reference. However, an even more attractive option is to take advantage of the superior stability already demonstrated by the simple iodine-stabilized Nd:YAG laser. As shown by the second case in Fig. 1(b) and the implementation scheme in Fig. 1(c), one comb line at each end of the comb spectrum is stabilized. As shown in Fig. 3(b), the 1064-nm comb line is phase locked to the cw laser, leading to a rms noise of $\sim 1 \mathrm{~Hz}$ in the beat note at

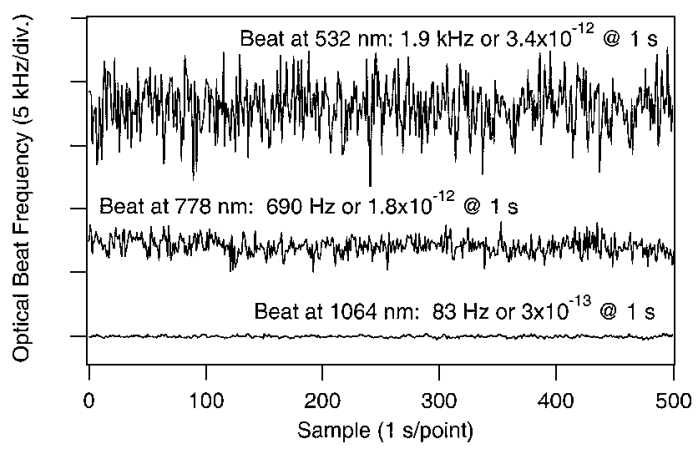

Fig. 2. Optical beat noise versus color (frequency offset from $1064 \mathrm{~nm}$ ). Std. Dev., standard deviation. 

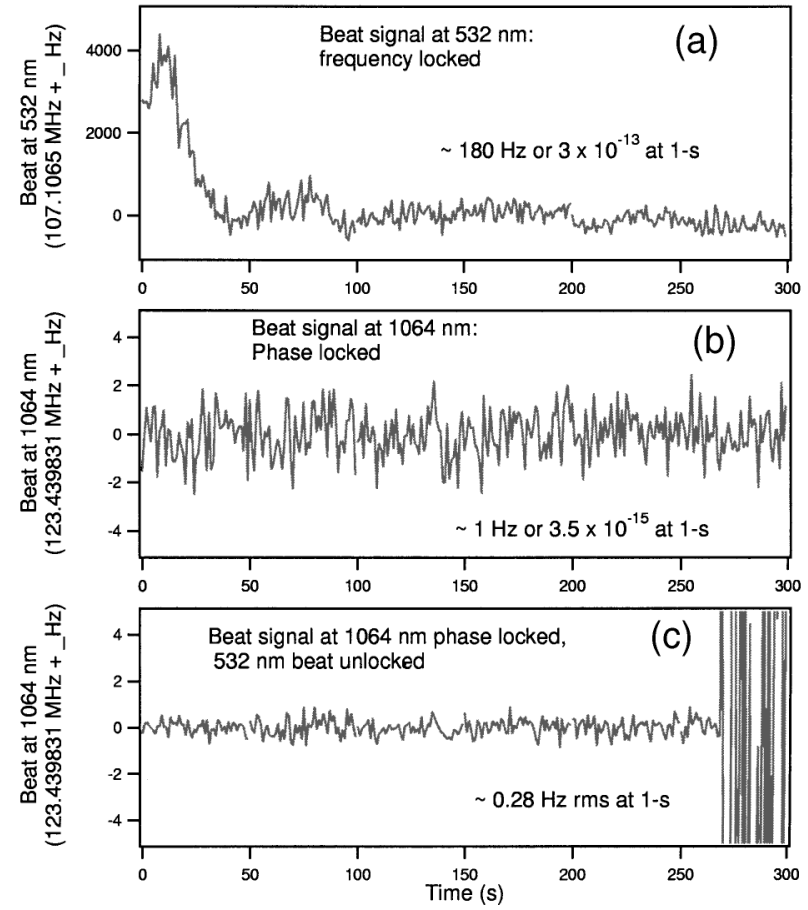

Fig. 3. Simultaneous stabilization of two comb lines. (a) A comb line at $532 \mathrm{~nm}$ is frequency locked to the $\mathrm{cw}$ laser, and their beat is counted with a 1-s gate time. (b) At the same time a comb line at $1064 \mathrm{~nm}$ is phase locked to the cw laser, with a standard deviation of $\sim 1 \mathrm{~Hz}$. (c) Only the 1064-nm beat is stabilized (phase locked), with a resultant frequency standard deviation of $280 \mathrm{mHz}$.

1-s averaging time. Figure 3(a) shows the 532-nm comb line being frequency locked to the cw laser; the resultant fluctuation of the beat frequency is $\sim 180 \mathrm{~Hz}$ (out of $564 \mathrm{THz}$ ) at $1 \mathrm{~s}$, aside from the initial $4-\mathrm{kHz}$ transient when the frequency-to-voltage converter was first activated. The reason that stabilization at $532 \mathrm{~nm}$ is not based on PLL is that there are still remaining issues in terms of the control orthogonalization between the two laser PZT's. Movement of each comb line receives contributions from fluctuations in both cavity length and dispersion. It is therefore not exactly appropriate to use the translating PZT for stabilization of one comb line and the tilting PZT for the other. This effect can be seen in the data of Fig. 3(c), where the phase-lock result at $1064 \mathrm{~nm}$ is improved by a factor of $\sim 4$ ( $\mathrm{rms}$ frequency noise of $0.28 \mathrm{~Hz}$ at $1 \mathrm{~s}$ ) when the lock loop at $532 \mathrm{~nm}$ is turned off. We note that the measured PLL data were obtained directly from the beat signal (without prescaling) with an independent rf tracking oscillator located outside the PLL loop.

With the stability of the optical frequency comb currently limited by the microwave reference used for phase locking $\Delta$, direct stabilization of comb components with stable optical references appears to hold great promise. To improve the frequency control, we need to explore the issue of orthogonal control of the two PZT's. Suppose that we denote the two beat error signals, at 1064 and $532 \mathrm{~nm}$, $\delta f_{1064}$ and $\delta f_{532}$, respectively. Let the pivot point of the tilting mirror (controlled by the tilting PZT) be centered at $f_{0}$, between the two references $f_{1064}$ and $f_{532}$. We denote $x=\left(f_{0}-f_{1064}\right) / f_{1064}$. Then one finds the control signal fed to the tilting PZT should have the form $\left(\delta f_{532}-2 \times \delta f_{1064}\right) /(1+x)$, whereas the control signal fed to the translating PZT is $\left[x \times \delta f_{532}+(1-x) \times \delta f_{1064}\right] /(1+x)$. The physical meaning of these relations is clear: The difference between $\delta f_{532}$ and $2 \times \delta f_{1064}$ arises solely from the change in mode spacing, whereas the weighted average (normalized common mode) of the two error signals is related to the change in cavity length. Electronic implementation of this orthogonalization procedure is under way. At the present stage, all the comb lines (covering one octave of the optical frequency spectrum) are already stable (relative to the Nd:YAG laser, $5 \times 10^{-14}$ ) to a level of 1 to $100 \mathrm{~Hz}$ at $1 \mathrm{~s}$. With control orthogonalization, we expect that every comb line will be phase locked to the $\mathrm{cw}$ reference below $1 \mathrm{~Hz}$. We would need some other ultrastable lasers for an independent check. Because we now can generate a stable microwave frequency directly from a stable laser, another important experiment will be a comparison of the stabilized $\Delta$ against an ultrastable microwave source, paving the way for the eventual realization of an optical atomic clock.

We thank D. J. Jones and S. T. Cundiff for helpful discussions. The research at JILA is supported by the National Institute of Standards and Technology (NIST), NASA, and the National Science Foundation. J. Ye's e-mail address is ye@jila.colorado.edu.

*Present address, Time and Frequency Division, NIST, 325 Broadway, Boulder, Colorado.

\section{References}

1. Th. Udem, J. Reichert, R. Holzwarth, and T. W. Hänsch, Phys. Rev. Lett. 82, 3568 (1999); Opt. Lett. 24, 881 (1999).

2. S. A. Diddams, D. J. Jones, J. Ye, S. T. Cundiff, J. L. Hall, J. K. Ranka, R. S. Windeler, R. Holzwarth, Th. Udem, and T. W. Hänsch, Phys. Rev. Lett. 84, 5102 (2000).

3. D. J. Jones, S. A. Diddams, J. K. Ranka, A. Stentz, R. S. Windeler, J. L. Hall, and S. T. Cundiff, Science 288, 635 (2000).

4. R. J. Rafac, B. C. Young, J. A. Beall, W. M. Itano, D. J. Wineland, and J. C. Bergquist, Phys. Rev. Lett. 85, 2462 (2000); J. E. Bernard, A. A. Madej, L. Marmet, B. G. Whitford, K. J. Siemsen, and S. Cundy, Phys. Rev. Lett. 82, 3228 (1999); H. Schnatz, B. Lipphardt, J. Helmcke, F. Riehle, and G. Zinner, Phys. Rev. Lett. 76, 18 (1996); C. W. Oates, E. A. Curtis, and L. Hollberg, Opt. Lett. 25, 1603 (2000).

5. F. L. Walls and A. DeMarchi, IEEE Trans. Instrum. Meas. 24, 210 (1975).

6. J. Reichert, R. Holzwarth, Th. Udem, and T. W. Hansch, Opt. Commun. 172, 59 (1999).

7. J. Ranka, R. Windeler, and A. Stentz, Opt. Lett. 25, 25 (2000).

8. J. L. Hall, L. S. Ma, M. Taubman, B. Tiemann, F. L. Hong, O. Pfister, and J. Ye, IEEE Trans. Instrum. Meas. 48, 583 (1999); J. Ye, L. Robertson, S. Picard, L. S. Ma, and J. L. Hall, IEEE Trans. Instrum. Meas. 48, 544 (1999). 